\title{
Inversão de dados de FTG e magnetometria do depósito N4WS utilizando dados petrofísicos coletados nos testemunhos de sondagem. - Carajás - Brasil
}

João Paulo Gomes de Souza (IGC - UNB), Adalene Moreira Silva (IGC - UNB), Catarina L. Toledo (IGC-UNB) e Luciano Mozer de Assis (DIPF - VALE)

Copyright 2016, SBGf - Sociedade Brasileira de Geofísica

Este texto foi preparado para a apresentação no VII Simpósio Brasileiro de Geofísica, Ouro Preto, 25 a 27 de outubro de 2016. Seu conteúdo foi revisado pelo Comitê Técnico do VII SimBGf, mas não necessariamente representa a opinião da SBGf ou de seus associados. É proibida a reproducãa total ou parcial deste material para propósitos comerciais sem prévia autorização da SBGt.

\section{Resumo}

Foi realizado um levantamento aéreo de FTG e magnetometria sobre 0 depósito de ferro N4WS, localizado na Província Mineral de Carajás, para que se pudesse identificar a resposta geofísica dos depósitos de ferro associados aos hematititos friáveis existente na região. Alem disso foram coletadas informações petrofísicas, susceptibilidade magnética e densidade, nos furos exploratórios realizados no depósito. A partir dos dados aéreos e dos dados petrofísicos, foi realizada uma inversão dos dados de FTG e magnéticos, utilizando as informações dos furos como modelo inicial. O resultado da inversão mostra que os dados de FTG estão correlacionados com a mineralização superficial de ferro (hematitito friável) uma vez que esse litotipo apresenta densidade média de $3.13 \mathrm{~g} / \mathrm{cm}^{3}$. As anomalias magnéticas observadas sobre o depósito, representam os jaspilitos em profundidade (protominério).

\section{Introdução}

O alvo N4SW está localizado na Província Mineral de Carajás (CMP), a oeste da mina de ferro do N5 e sudoeste da mina de ferro do N4. Com o intuito de se obter a resposta geofísica dos depósitos de ferro do N5 e N4, e procurar por novos alvos, foi realizado um levantamento aéreo de Full Tensor Gravity Gradiometry (FTG) e magnetometria. O levantamento foi realizado com linhas na direção $\mathrm{N} 60^{\circ} \mathrm{W}$, com espaçamento de 150 metros entre as linhas e altura de voo de 150 metros. Conforme Assis (2013) a direção das linhas de voo foi planejada para que houvesse a menor influência possível da topografia nos dados de FTG (Braga, 2009). Ao todo foram levantados $276 \mathrm{~km}^{2}$ em 76 perfis geofísicos. O depósito do N4WS fica na porção NW do levantamento (Fig 01) e é caracterizado por um forte alto topográfico na região central da área de estudo.

Sobre o depósito N4WS os dados de FTG revelaram uma anomalia na componente vertical (Tzz) com aproximadamente $5,8 \mathrm{~km}$ e 70 Eo de amplitude (Fig 02). Assis (2013) mostrou que a anomalia de FTG nessa componente ressalta os depósitos de ferro, enquanto as outras componentes tensoriais (Txx, Txy, Txz, Tyy e Tyz) ressaltam feições que podem ser utilizadas para 0 mapeamento do arcabouço estrutural da região.

O levantamento de magnetometria (Fig 03) mostrou duas fortes anomalias magnéticas, denominadas $A N$ e AS, tendo possivelmente como fonte magnética os jaspilitos presentes na região. Assis (2013) já chamava a atenção para o fato que a região mineralizada não possuir resposta magnética, uma vez que a magnetita e a martita estão associadas ao protominério, enquanto a hematita está correlacionada a mineralização.

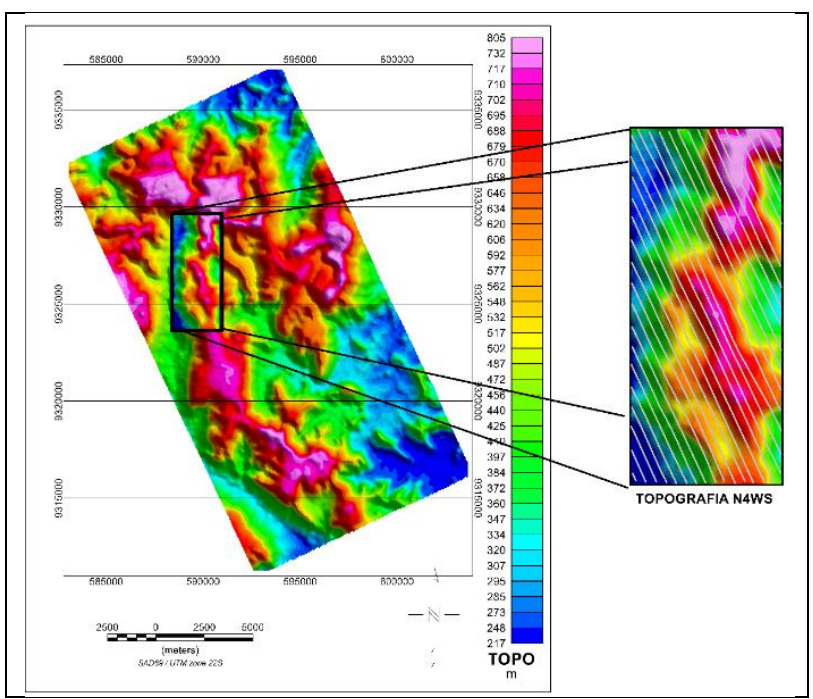

Figura 01: Topografia do levantamento de FTG e magnetometria, destacando a região de estudo, N4WS, e a sua topografia. A linhas cinzas na figura identificam as linhas de voo executadas.

Para a caracterização dos litotipos e determinação das principais fontes anômalas, foram realizados levantamentos petrofísicos de densidade e susceptibilidade magnética nos furos de sondagem realizados no alvo N4WS, sendo realizada uma medida a cada um metro. Ao todo foram analisados 1948 metros de sondagem.

O hematitito compacto $(\mathrm{HC})$ foi o litotipo mais denso, com média em torno de $3,61 \mathrm{~g} / \mathrm{cm}^{3}$, seguindo pelos jaspilitos (JP) $3,18 \mathrm{~g} / \mathrm{cm}^{3}$, formações ferríferas manganíferas $\left(3,17 \mathrm{~g} / \mathrm{cm}^{3}\right)$, hematititos manganesiferos $\left(3,13 \mathrm{~g} / \mathrm{cm}^{3}\right)$ e hematititos friáveis $(\mathrm{HF}), 3,12 \mathrm{~g} / \mathrm{cm}^{3}$. O único litotipo magnético presente na região é o jaspilito (JP) com susceptibilidade magnética média de $0,23 \mathrm{SI}$, os outros litotipos presentes na região tem susceptibilidade magnética menor que $0,01 \mathrm{SI}$ e podem ser considerados como não magnéticos.

A partir dos dados petrofísicos obtidos, foram criados modelos geofísicos de distribuição de propriedade física (densidade e susceptibilidade magnética) em 3D. Esses modelos foram utilizados como modelos iniciais durante o processo de inversão dos dados geofísicos. 
A proposta desse trabalho é avaliar os processos de inversão dos dados magnéticos e de FTG, utilizando como modelo inicial os dados petrofísicos, comparando os resultados obtidos com os modelos geológicos sugeridos para o alvo. Evidente que a utilização de um modelo inicial com a geologia diminuirá a incerteza do modelo geofísico e com isso espera-se que possamos determinar a existência ou não de fontes anômalas profundas ou não investigadas por sondagens.

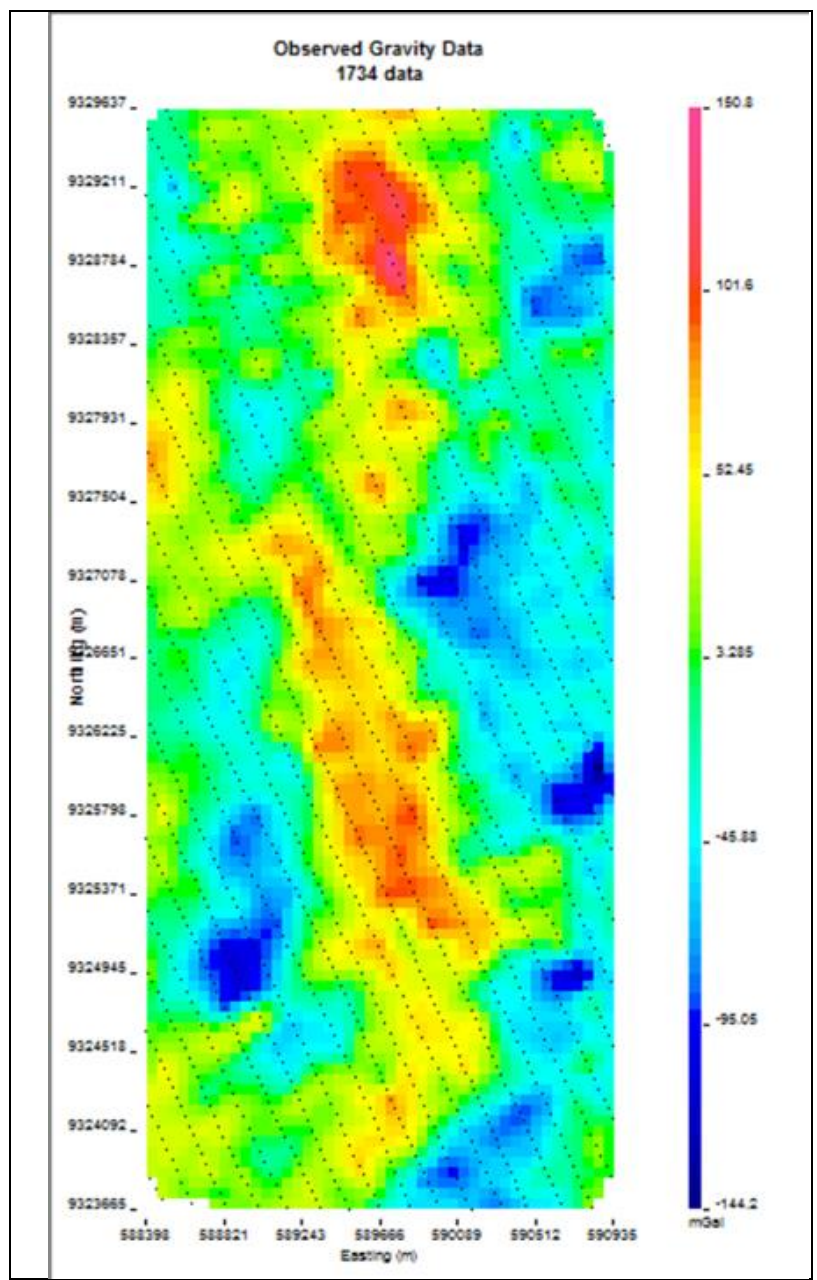

Figura 02: Anomalia de FTG, componente Tzz e correção de terreno de $2,67 \mathrm{~g} / \mathrm{cm}^{3}$. Os pontos pretos representam as estações de medidas.

\section{Metodologia}

Para a realização desse trabalho foi proposta a seguinte metodologia:

- Correção de terreno;

- Determinação da direção de magnetização;

- Construção do modelo geológico inicial;

- Inversão dos dados geofísicos;

Para a correção de terreno dos dados de FTG foi utilizada a metodologia sugerida por FitzGerald e Patterson (2013). Atraves desse método a densidade que melhor representar a correlação entre a topografia e os dados de FTG irá se ajustar melhor a equação abaixo:

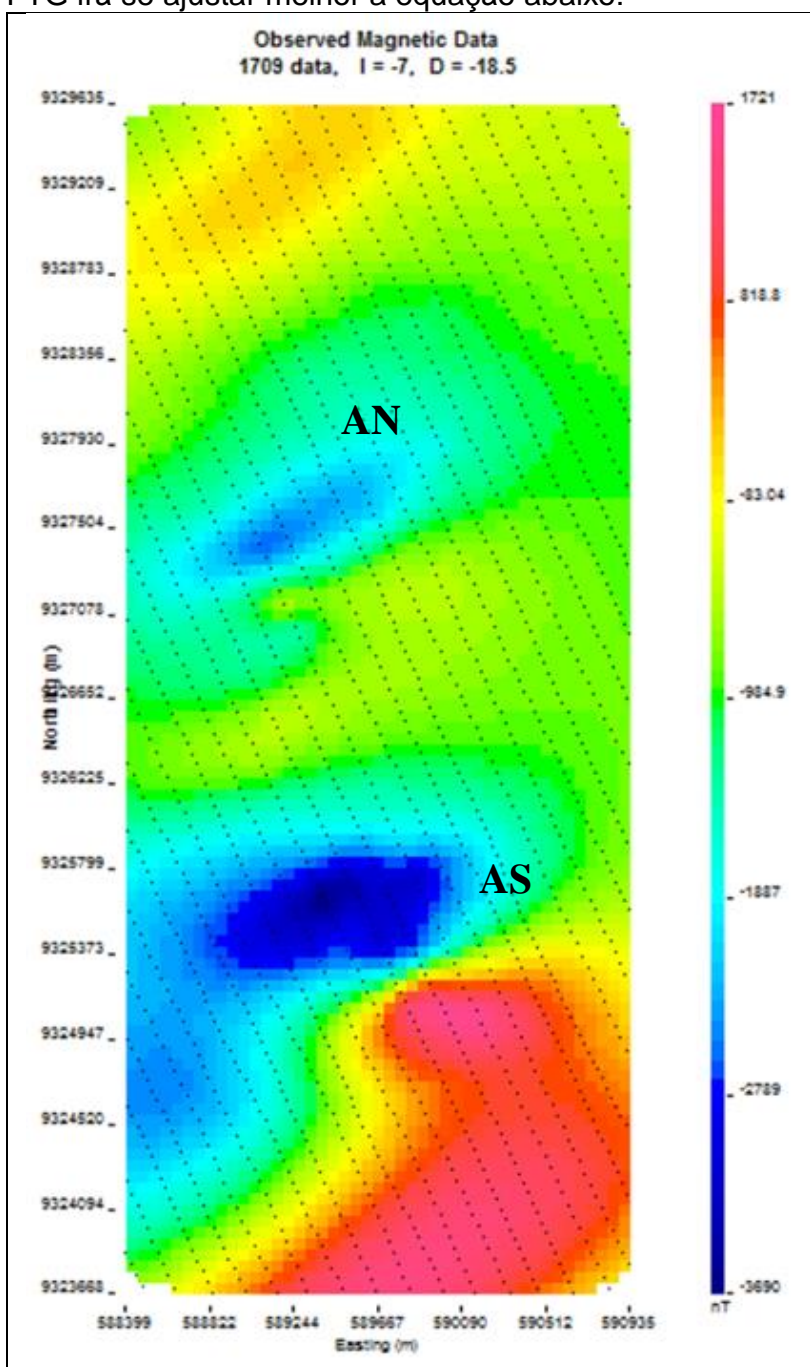

Figura 03: Anomalia de magnética, campo magnético total, mostrando as anomalias AN e AS. Os pontos pretos representam as estações de medidas

$$
\sum(F a-\rho * T C)^{2}=0
$$

onde Fa é anomalia Free-air medida, TC é a correção de de terreno e $\rho$ é a densidade analisada. Para os dados do alvo N4WS a densidade que melhor se ajusta é de $2,819 \mathrm{~g} / \mathrm{cm}^{3}$ (Fig 04). Esse valor é inferior a densidade do pacote mineralizado que é de $3,04 \mathrm{~g} / \mathrm{cm}^{3}$.

Para a determinação da direção de magnetização foi utilizada a metodologia sugerida por Dannemiller e LI (2006) utilizando a redução ao polo para baixas latitudes (Li, 2008). Essa foi escolhida, pois, já foi utilizada com sucesso por Souza et al (2015) na mesma região da CMP.

O método desenvolvido por Dannemiller e Li (2006) utiliza a correlação cruzada entre o gradiente vertical da anomalia magnética reduzida ao polo e o gradiente total da anomalia também reduzida ao polo. 
Como a transformação de redução ao polo pode ser tornar instável em regiões de baixa latitude magnética, o campo total transformado (RTP-L) foi comparado com o gradiente total e com o sinal analítico da integração vertical do campo magnético anômalo (ASVI, Paine et al, 2001). A tabela1 mostra a direção de magnetização obtida pelo método de Dannemiller e Li.

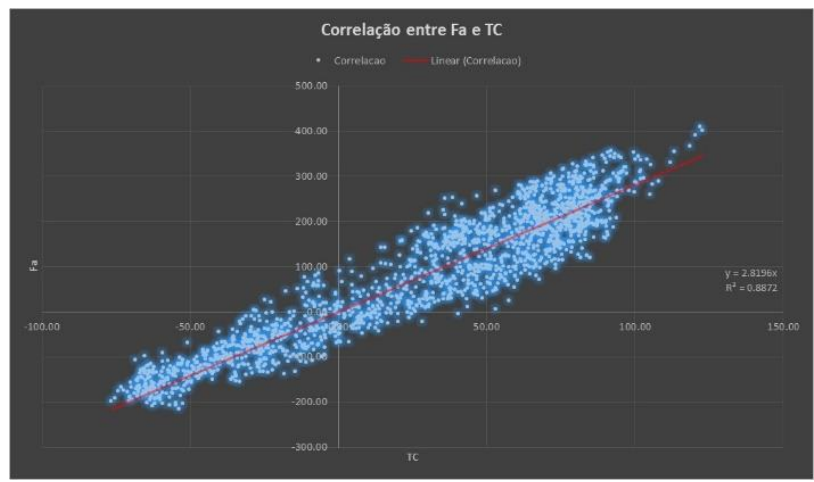

Figura 04: Ajuste dos mínimos quadrados entre os dados de anomalia Free-air e a correção de terreno. É possível ver que a densidade que melhor se ajusta é $2,819 \mathrm{~g} / \mathrm{cm}^{3}$.

Tabela 1: Valores de remanência, direção total e da razão de Konigsberge para as anomalias AN e AS.

\begin{tabular}{|c|c|c|c|c|c|}
\hline Anomalia & \multicolumn{2}{|c|}{ Remanência } & \multicolumn{2}{c|}{ Direção total } & Q \\
\hline \multirow{2}{*}{ AN } & Inclinação & 820 & Inclinação & $52^{\circ}$ & \multirow{2}{*}{1.5} \\
\cline { 2 - 5 } & Declinação & 390 & Declinação & 3480 & \\
\hline \multirow{2}{*}{ AS } & Inclinação & 680 & Inclinação & 550 & \multirow{2}{*}{3} \\
\cline { 2 - 5 } & Declinação & 80 & Declinação & 3560 & \\
\hline
\end{tabular}

Para a inversão dos dados geofísicos do alvo N4WS foram construídos dois modelos litológicos iniciais, uma para a gravimetria e outro para a magnetometria.

Os dois modelos tiveram como base os dados dos furos de sondagens realizados no alvo. A cada litotipo interceptado pelas sondagens foi dada a média dos valores de densidade e de susceptibilidade obtido. Os dados de densidade foram retirados de Assis (2013). Já os dados de densidade foram coletados nos furos de sondagem. As figuras 05 e 06 mostram a distribuição dos dados de densidade e susceptibilidade nos furos.

Para a inversão dos dados geofísicos foram utilizados dois códigos diferentes. Para os dados de FTG foi utilizado o GGINV3D (Li e Oldenburg, 2001). Para os dados de magnetometria foi utilizado o código MAGINV3D (Li e Oldenburg, 1996). Como o MAGINV3D não trabalha com a magnetização remanescente foi necessário determinar a direção total da magnetização.

Em ambos os casos o mesh utilizado foi de $50 \mathrm{~m} 50 \mathrm{~m} x$ $25 \mathrm{~m}(X Y Z)$, e erro utilizado foi de $5 \%$ do desvio padrão do dado de potencial, no caso da magnetometria foi utilizado um erro de 52nT e de FTG foi utilizado o erro de 2.5Eo. Para as duas inversões os dados topográficos utilizados foram retirados do levantamento aéreo. Em todos os casos o número de medidas utilizadas para a inversão foram de 1709, distribuídas ao longo das linhas de aquisição.
A escolha do parâmetro de regularização para os dados de magnetometria foi feita de forma automática pelo código MAGINV3D, já o código GGINV3D permite que o usuário realize a escolha do melhor parâmetro de regularização. Essa escolha é realizada através da curva L (Li e Oldenburg, 2007). Para a confecção dessa curva são realizadas várias inversões com diferentes valores de parâmetro de regularização e então é feito um gráfico do misfit pela norma de cada inversão. O valor mais próximo da inflexão da curva pode ser adotado como o melhor valor para o parâmetro de regularização.

Para o dado de FTG em questão foram realizadas 10 inversões com o maior parâmetro de regularização igual a 1000 e o menor parâmetro foi 0.000001 .

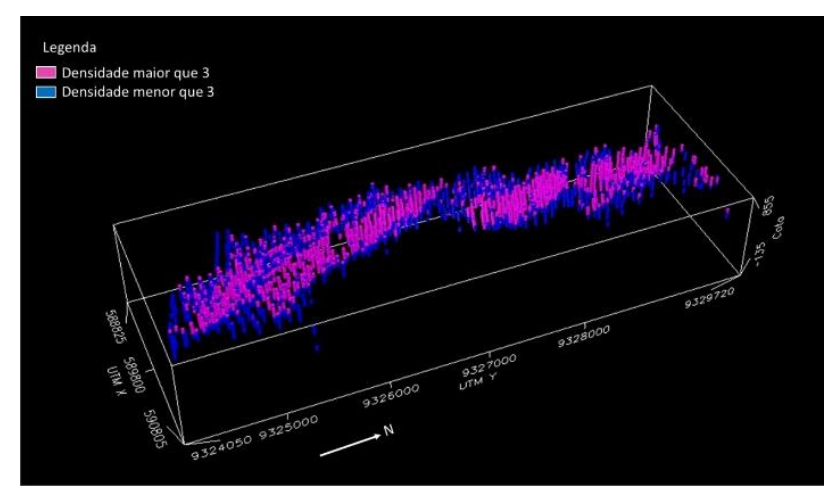

Figura 05: Distribuição dos valores de densidade nos furos de sondagem. A cor azul representa valores de densidade menores que $3 \mathrm{~g} / \mathrm{cm}^{3}$, enquanto os valores maiores que $3 \mathrm{~g} / \mathrm{cm}^{3}$ estão representados pela cor magenta.

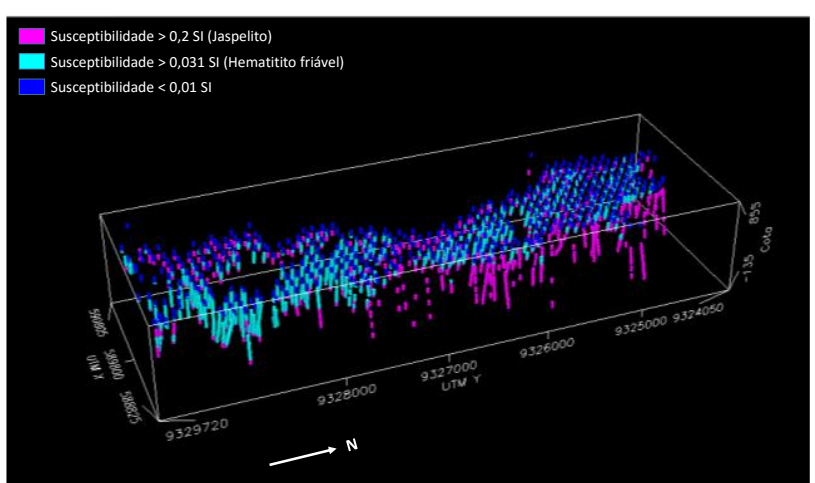

Figura 06: Distribuição dos valores de susceptibilidade magnética nos furos de sondagem. A cor azul escura representa valores de susceptibilidade menores que 0,01 Sl, a cor azul clara representa valores de susceptibilidade maiores que 0,01 SI e menores que 0,2 SI. A cor magenta está correlacionada a valores de susceptibilidade maiores que 0,2 SI (jaspilito).

\section{Resultados}

\section{Dados Gravimétricos}

A figura 07 mostra o resultado da inversão para os dados de gravimetria a partir do modelo inicial de densidade.

Como pode ser visto na figura 07 a anomalia causada pelo modelo de densidade obtido após a inversão dos dados, 
apresentou boa correspondência com os dados medidos. O bloco de densidade obtido obteve com valor máximo de densidade $0.517 \mathrm{~g} / \mathrm{cm}^{3}$, considerando a correção de terreno $\left(2,819 \mathrm{~g} / \mathrm{cm}^{3}\right)$ esse valor em termos absolutos é equivalente a $3,336 \mathrm{~g} / \mathrm{cm}^{3}$, próximo aos valores encontrados para os hematititos friáveis (HF). A figura 08 mostra o bloco de densidade obtido para a região investigada.

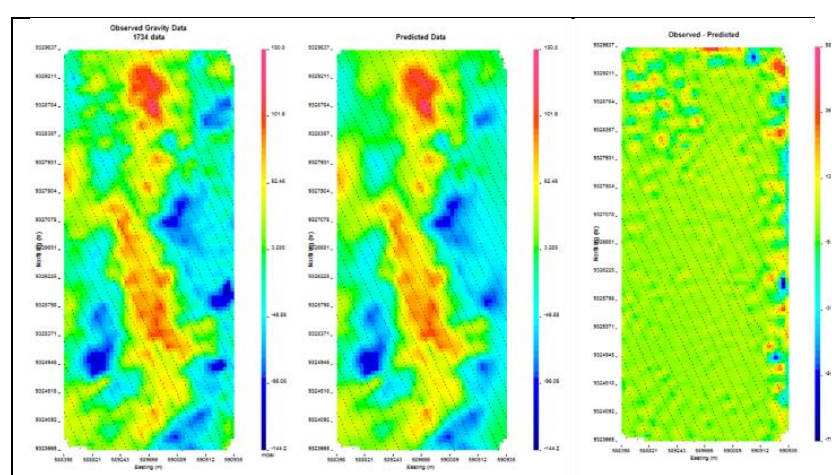

Figura 07: Os dados medidos podem ser vistos na parte esquerda da figura, enquanto o predicted data pode ser visto na parte central. Os resíduos podem ser observados na parte direita da figura.

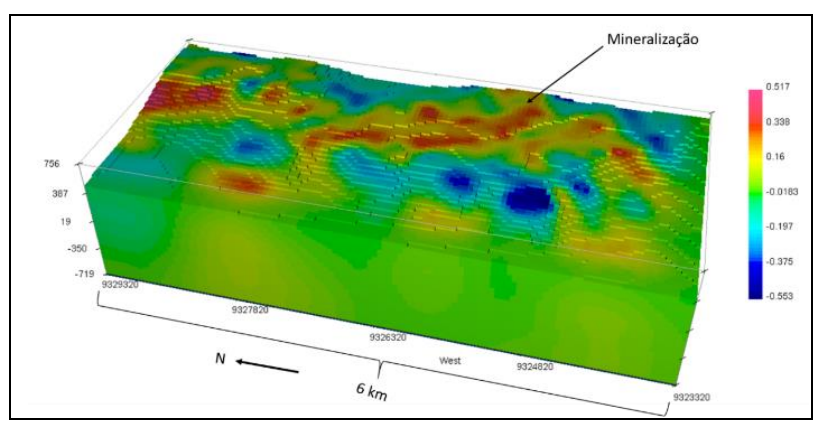

Figura 08: Os dados medidos podem ser vistos na parte esquerda da figura, enquanto o predicted data pode ser visto na parte central. Os resíduos podem ser observados na parte direita da figura.

Para comparar os resultados obtidos pela inversão com os furos de sondagem foram realizadas seções E-W, a figura 09 mostra o resultado para a seção $A B$. Nessa figura é possível ver que a região mais densa na seção vertical corresponde a zona com hematitito friável $\left(3,12 \mathrm{~g} / \mathrm{cm}^{3}\right)$, para os litotipos com densidade menor que $3 \mathrm{~g} / \mathrm{cm}^{3}$ não foi recuperado nenhum corpo.

\section{Dados de magnetometria}

A figura 10 mostra o resultado da inversão para os dados de magnetometria a partir do modelo inicial de susceptibilidade.

Como pode ser visto na figura 10 as anomalias magnéticas causadas pelo modelo de susceptibilidade obtido após a inversão dos dados magnéticos, apresentou boa correspondência com os dados medidos. O bloco de susceptibilidade magnética obtido, obteve com valor máximo de densidade próximo a 1.16SI e duas fontes magnéticas profundas (AN e AS). Esses valores são superiores aos valores de susceptibilidade encontrados nos testemunhos de sondagem para os jaspilitos. A figura 11 mostra o bloco de susceptibilidade obtido para a região investigada.

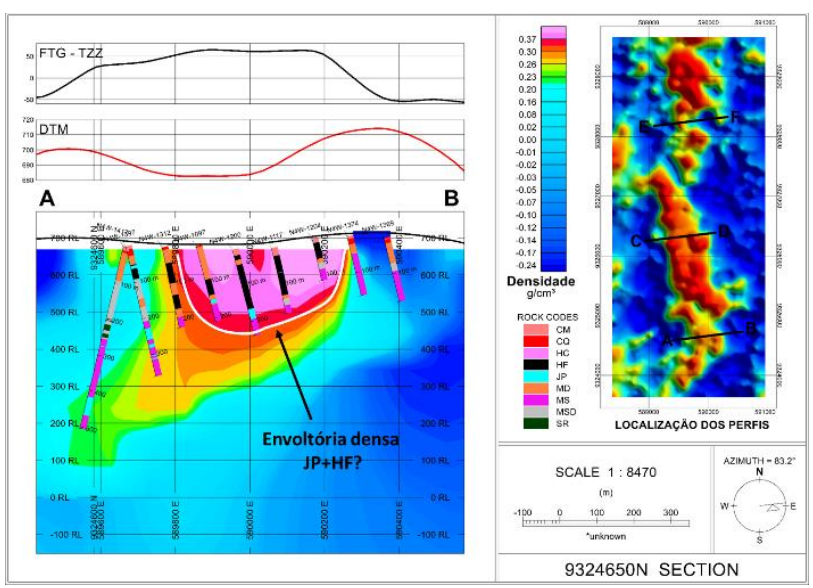

Figura 09: Seção $A B(E-W)$ mostrando os furos executados na seção e os corpos recuperados pela inversão dos dados de FTG.

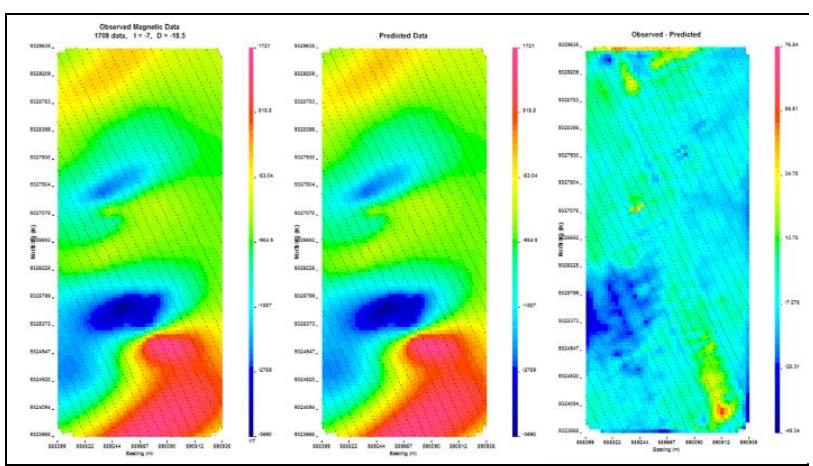

Figura 10: Os dados medidos podem ser vistos na parte esquerda da figura, enquanto o predicted data pode ser visto na parte central. Os resíduos podem ser observados na parte direita da figura.

Como realizado para o modelo gravimétrico, foram realizadas várias seções e os resultados dos modelos foram comparados com os furos exploratório. A figura 12 mostra uma seção NW-SE cortando as duas anomalias observadas nos dados.

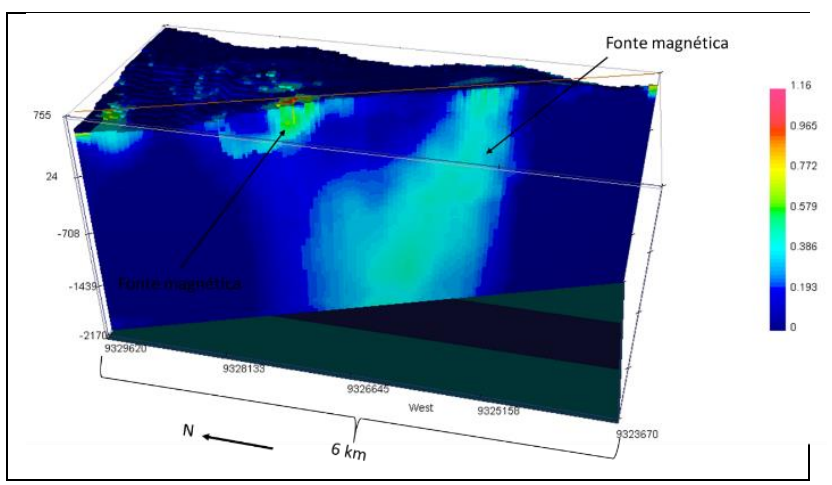

Figura 11: Bloco de susceptibilidade obtido para a região do N4WS com as duas fontes magnéticas recuperadas. $A$ 
fonte mais rasa é atribuída a anomalia $A N$ e a fonte profunda é atribuída a anomalia $A S$.

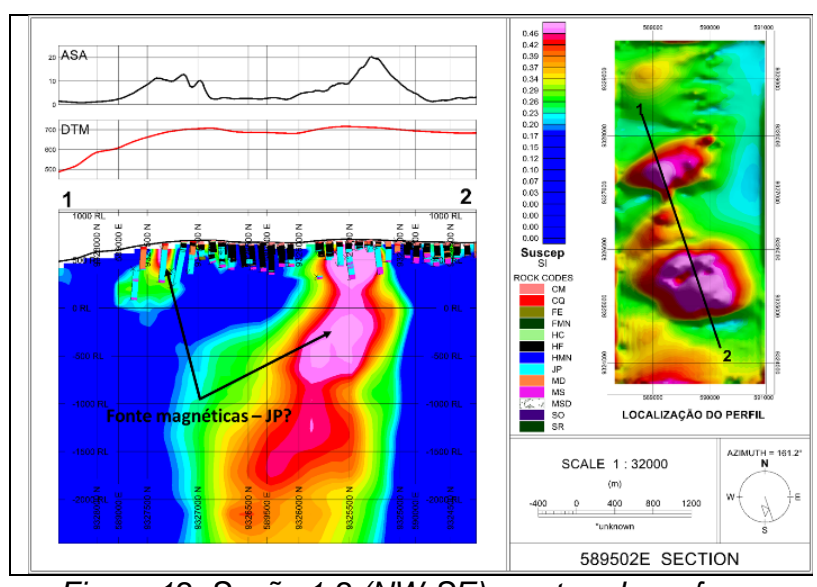

Figura 12: Seção 1-2 (NW-SE) mostrando os furos executados na seção e os corpos recuperados pela inversão dos dados de magnetometria. Nos furos os litotipos representados pela cor cian estão correlacionados aos jaspilitos.

\section{Discussão e Conclusões}

O modelo de densidade 3D obtido após a inversão dos dados da componente vertical (Tzz) dos dados de FTG sobre o depósito N4SW, mostrou que o corpo recuperado é superficial com densidade variando entre $3 \mathrm{~g} / \mathrm{cm}^{3}$ e $3.13 \mathrm{~g} / \mathrm{cm}^{3}$ e sua disposição espacial é coincidente com a região mineralizada. No modelo não é possível observar fontes anômalas em profundidade. Quando comparamos - modelo obtido com os resultados dos furos de sondagem, percebemos que houve uma boa aderência.

Observando o modelo de susceptibilidade em 3D, observamos que as duas fontes magnéticas observadas no campo magnético total apresentam significativas fontes anômalas.

A anomalia AN apresenta um corpo superficial, enquanto a anomalia AS se encontra mais profunda. O modelo recuperado não mostra nenhuma correlação direta com a mineralização. Esse resultado já era esperado, pois a mineralização, hematitito friável, não apresenta magnetismo e apenas os jaspilitos são magnéticos.

Apesar dos jaspílitos se apresentarem densos, $3,18 \mathrm{~g} / \mathrm{cm}^{3}$, o modelo de densidade não mostra nenhuma influência de fontes profundas coincidentes com os corpos magnéticos, somente corpos rasos correlacionados com a mineralização.

Com isso podemos concluir que a inversão de dados de FTG utilizando os dados de densidade como modelo inicial, se mostrou eficiente para delimitar e verificar a continuidade em profundidade dos corpos mineralizados, enquanto o modelo de susceptibilidade foi capaz de recuperar as fontes anômalas em profundidade, atribuídas aos jaspilitos. Um fato que devemos levar em consideração é que nos dados dos furos utilizados, haviam muito mais dados de densidade relacionados as fontes anômalas do que dados magnéticos, uma vez que as sondagens se limitaram apenas a porção mineralizada e sem magnetismo.

\section{Agradecimentos}

Os autores agradecem a companhia Vale S.A por autorizar a publicação do trabalho e a utilização dos softwares de inversão e a FUNAPE pela bolsa concedida durante a pesquisa

\section{Referências}

Assis, L.M., 2013, Geração de Modelo de Exploração para - Minério de Ferro da Província Mineral de Carajás Através da Integração de Dados Multifonte, Dissertação de Mestrado em Geologia, nำ310, Instituto de Geociência da Universidade de Brasília, 154p

Braga, M. A., Galbiatti, H., Carlos, D. U., Sousa, R. R., Almeida, T., 2009, Planejamento e controle de qualidade de dados de gradiometria gravimétrica 3D-FTG: Revista Brasileira de Geofísica, v.27(3), p. 447-457.

Dannemiller, N. and Li, Y, 2006, A new method for determination of magnetization direction, Geophysics, 71, 6, L69-L73.

FriztGerald, D and Paterson, R.,2013, Getting the best value from gravity gradiometry, Society of Exploration Geophysicist Annual Meeting, Houston

Li, Y. and Oldenburg, D. W., 1996, 3-D inversion of magnetic data: Geophysics, 61, no. 02, 394-408.

Li, Y., 2001, 3D inversion of gravity gradiometry data, Expanded Abstract, 71th Ann. Int. Mtg, Soc. Explor. Geophys., Tulsa

Li, X., 2008, Magnetic reduction-to-the-pole at low latitudes: observations and considerations. The Leading Edge 27, 990-1002

Paine, J., Haederle, M., and Flis, M., 2001, Using transformed TMI data to invert for remanently magnetized bodies, Exploration Geophysics, 32, 238-242.

Souza, J. P. G., Leão-Santos, M. H., Freitas, C. O., Matos, F. M. V., Carvalho, D. L., 2015, Applicability of Standard Euler deconvolution, modeling, and amplitude magnetic data inversion in Greenfield programs: The Leite target case study, Carajás Mineral Province, Brazil, Interpretation, 3, T131 - T141. doi:10.1190/INT-20140192.1 